\title{
Evaluation by simulation of clinical trial designs for evaluation of treatment during a viral haemorrhagic fever outbreak
}

\author{
Pauline Manchon ${ }^{1,2^{*}+}$ (D), Drifa Belhadi ${ }^{1,2,3 \dagger}$, France Mentré ${ }^{1,2,3}$ and Cédric Laouénan ${ }^{1,2,3}$
}

\begin{abstract}
Background: Viral haemorrhagic fevers are characterized by irregular outbreaks with high mortality rate. Difficulties arise when implementing therapeutic trials in this context. The outbreak duration is hard to predict and can be short compared to delays of trial launch and number of subject needed (NSN) recruitment. Our objectives were to compare, using clinical trial simulation, different trial designs for experimental treatment evaluation in various outbreak scenarios.

Methods: Four type of designs were compared: fixed or group-sequential, each being single- or two-arm. The primary outcome was 14-day survival rate. For single-arm designs, results were compared to a pre-trial historical survival rate $\mathrm{p}_{\mathrm{H}}$. Treatments efficacy was evaluated by one-sided tests of proportion (fixed designs) and Whitehead triangular tests (group-sequential designs) with type---error $=0.025$. Both survival rates in the control arm $p_{C}$ and survival rate differences $\Delta$ (including 0 ) varied. Three specific cases were considered: "standard" (fixed $p_{C}$, reaching NSN for fixed designs and maximum sample size $\mathrm{N}_{\text {Max }}$ for group-sequential designs); "changing with time" (increased $p_{C}$ over time); "stopping of recruitment" (epidemic ends). We calculated the proportion of simulated trials showing treatment efficacy, with $\mathrm{K}=93,639$ simulated trials to get a type-l-error $\mathrm{Pl}_{95 \%}$ of $[0.024 ; 0.026]$.

Results: Under $\mathrm{H}_{0}(\Delta=0)$, for the "standard" case, the type---error was maintained regardless of trial designs. For "changing with time" case, when $p_{C}>p_{H}$, type-l-error was inflated, and when $p_{C}<p_{H}$ it decreased. Wrong conclusions were more often observed for single-arm designs due to an increase of $\Delta$ over time. Under $\mathrm{H}_{1}(\Delta=+0.2)$, for the "standard" case, the power was similar between single- and two-arm designs when $\mathrm{p}_{\mathrm{C}}=\mathrm{p}_{\mathrm{H}}$. For "stopping of recruitment" case, single-arm performed better than two-arm designs, and fixed designs reported higher power than group-sequential designs. A web R-Shiny application was developed.

(Continued on next page)
\end{abstract}

\footnotetext{
* Correspondence: pauline.manchon@aphp.fr

${ }^{\dagger}$ Pauline Manchon and Drifa Belhadi contributed equally to this work.

'INSERM, Centre d'Investigation clinique-Epidémiologie Clinique 1425, Hôpital Bichat, F-75018 Paris, France

${ }^{2}$ Département Epidémiologie Biostatistiques et Recherche Clinique, AP-HP, Hôpital Bichat, F-75018 Paris, France

Full list of author information is available at the end of the article
}

(c) The Author(s). 2021 Open Access This article is licensed under a Creative Commons Attribution 4.0 International License, which permits use, sharing, adaptation, distribution and reproduction in any medium or format, as long as you give appropriate credit to the original author(s) and the source, provide a link to the Creative Commons licence, and indicate if changes were made. The images or other third party material in this article are included in the article's Creative Commons licence, unless indicated otherwise in a credit line to the material. If material is not included in the article's Creative Commons licence and your intended use is not permitted by statutory regulation or exceeds the permitted use, you will need to obtain permission directly from the copyright holder. To view a copy of this licence, visit http://creativecommons.org/licenses/by/4.0/ The Creative Commons Public Domain Dedication waiver (http://creativecommons.org/publicdomain/zero/1.0/) applies to the data made available in this article, unless otherwise stated in a credit line to the data. 
(Continued from previous page)

Conclusions: At an outbreak beginning, group-sequential two-arm trials should be preferred, as the infected cases number increases allowing to conduct a strong randomized control trial. Group-sequential designs allow early termination of trials in cases of harmful experimental treatment. After the epidemic peak, fixed single-arm design should be preferred, as the cases number decreases but this assumes a high level of confidence on the pre-trial historical survival rate.

Keywords: Viral haemorrhagic fever outbreak, Clinical trial design, Simulation study

\section{Background}

Emerging infectious diseases such as influenza, cholera, coronavirus, varicella, meningitis and viral haemorrhagic fevers recently caused numerous outbreaks [1]. Since 2003, the coronavirus caused various outbreaks around the world. SARS-CoV-1 was first observed in Southeast Asia. In 2012, MERS-CoV caused a case-fatality rate of $37 \%$ in the Middle East [2]. Today SARS-CoV-2 is pandemic. Viral haemorrhagic fevers are among the most severe, including Crimean-Congo haemorrhagic fever, Ebola virus disease, Lassa fever and Marburg haemorrhagic fever, which are endemic in some areas of Africa, South America and Asia [3]. They are characterized by outbreaks with high mortality rate which occur irregularly and are hard to anticipate. The second largest outbreak of Ebola virus disease since the 2014-2016 one in West Africa, began in 2018 and ended in June 2020 in the Democratic Republic of the Congo [4].

Recently, a scoping review evaluated political, economic, administrative, regulatory, logistical, ethical and social (PEARLES) challenges associated with clinical research in the context of emergency epidemics [5]. The authors highlighted the challenges associated with the planning, conduct and dissemination of clinical research responses during an epidemic. They stressed the need for developing solutions to improve rapid clinical research deployment, delivery, and dissemination for future epidemics.

Difficulties often occur when implementing therapeutic trials in the context of viral haemorrhagic fever outbreak. Indeed, the duration of the outbreak is hard to predict and can be very short compared to the necessary delay to launch a trial and recruit the required number of patients. A reduced number of included patients impact the statistical power of the trial and can lead to study which cannot demonstrate a significant effect of their experimental treatment [6-8]. Evolution of the number of cases and case fatality rate during an outbreak period is also a dimension to be considered when designing a clinical trial in this context.

Moreover, the design of the study must be acceptable on the field by patients, healthcare workers and the global population. In the situation of an epidemic peak, local authorities and non-governmental organizations managing treatment centres may argue that randomization is hard to implement due to the high case-fatality rate and the reluctance to give a less beneficial treatment to part of the patients $[7,9,10]$. A singlearm design may seem to be best suited for those ethical aspects, its feasibility on the field and its acceptability by the population affected directly or indirectly by the disease. Nevertheless, non-comparative trials can lead to substantially biased results if the pre-trial survival rate is incorrect, indeed its evolution over time is not taken into account.

Another aspect to consider is the choice of the design: fixed or group-sequential. Indeed, group-sequential designs sometimes lead to reducing the duration of the trial especially in case of early stop for futility or efficacy of the experimental treatment [11]. At each interim analysis, the efficacy of the treatment is tested to decide if the recruitment has to continue or if the experimental treatment is already shown to be effective or not. Group-sequential designs are interesting in an epidemic peak of infectious disease, under emergency conditions and with different treatment candidates.

During the Ebola outbreak in West Africa, between December 2013 and May 2016, three antivirals (favipiravir, brincidofovir, TKM-13083), one cocktail of antibodies, and convalescent plasma were evaluated in five therapeutic trials in Guinea, Liberia and Sierra Leone $[6-8,12,13]$. These trials were implemented during the second phase of the outbreak, when the number of cases decreased, and none of them achieved the number of patients required. For four trials, the primary outcome was the mortality at 14 days after the inclusion. This outcome was chosen because it requires no invasive procedures or special equipment, and most of the time deaths from Ebola occur within 14 days. Only one used the mortality at 28 days and it was the only randomized trial with an adaptive scheme [6]. The four other trials were non-randomized and the comparison was established with the pre-trial mortality based on historical data, and three trials used a group-sequential design $[7,12$, 13]. More recently, a multi-arm multi-stage clinical trial was conducted with four investigational therapies for Ebola virus disease in the Democratic Republic of Congo, where, two of the four treatments compared were stopped based on interim analysis [14]. 
A simulation study by Cooper et al. [15] was conducted to investigate the choice of the study design in the context of the Ebola Virus disease. In particular, they worked on a multi-stage approach (MSA) comprising a single-arm phase II study followed by one or two phase III studies. They concluded that the MSA and group-sequential double-arm randomised trial led to substantially fewer deaths than a conventional two-arm randomised trial if the tested interventions were either highly effective or harmful. MSA was applied to design two clinical trials during the 2014-2015 West African Ebola outbreak [16].

The objectives of the present clinical trial simulation study was to compare different designs, during various outbreak scenarios to develop recommendations when designing a clinical trial for viral haemorrhagic fever. We considered several specific cases associated with the timeline of an outbreak. Indeed, at the beginning of an outbreak, standard cares are developed, which lead to increased survival rates. After the epidemic peak, it is more difficult to include patients in a trial due to a decreased number of cases. This crisis situation requires an accelerated research process and the study feasibility depends on the choice of the trial design. For this simulation study, we choose to compare four clinical trial designs: fixed single-arm, group-sequential single-arm, fixed two-arm and group-sequential two-arm designs. An online tool based on the results from this simulation study was also developed.

\section{Methods}

We performed clinical trial simulations to compare four designs (see Additional file 1): fixed single-arm trial (F1); group-sequential single-arm trial (S1); fixed two-arm trial (F2); and group-sequential two-arm trial (S2). A randomization 1:1 was considered for two-arm designs. The primary outcome of each simulated trial was the number of patients who survived at day 14 after inclusion. For single-arm designs, results were compared to a pre-trial historical survival rate.

Several outbreak scenarios were explored based on three scenario for the timeline of the outbreak and various values of control survival rate $\left(\mathrm{p}_{\mathrm{C}}\right)$ and efficacy of the treatment $(\Delta)$. A scenario was defined as the combination of one specific case of outbreak timeline, one control survival rate and one survival rate difference.

\section{Three specific cases of outbreak timeline (Fig. 1)}

The "standard" case corresponded to a trial launch at the beginning of the outbreak, i.e. a fixed $\mathrm{p}_{\mathrm{C}}$ and a number of subjects equal to the number of subjects needed (NSN) for fixed designs and the possibility to reach the maximum sample size $\left(\mathrm{N}_{\mathrm{Max}}\right)$, if needed for groupsequential trials.

The "changing with time" case was defined to mimic increased proportion of patients who survived in the control group over time during the outbreak $\left(\mathrm{p}_{\mathrm{C}}\right)$. An increase of +0.03 every 20 patients included was simulated, with a maximal total increase of 0.10 . This scenario

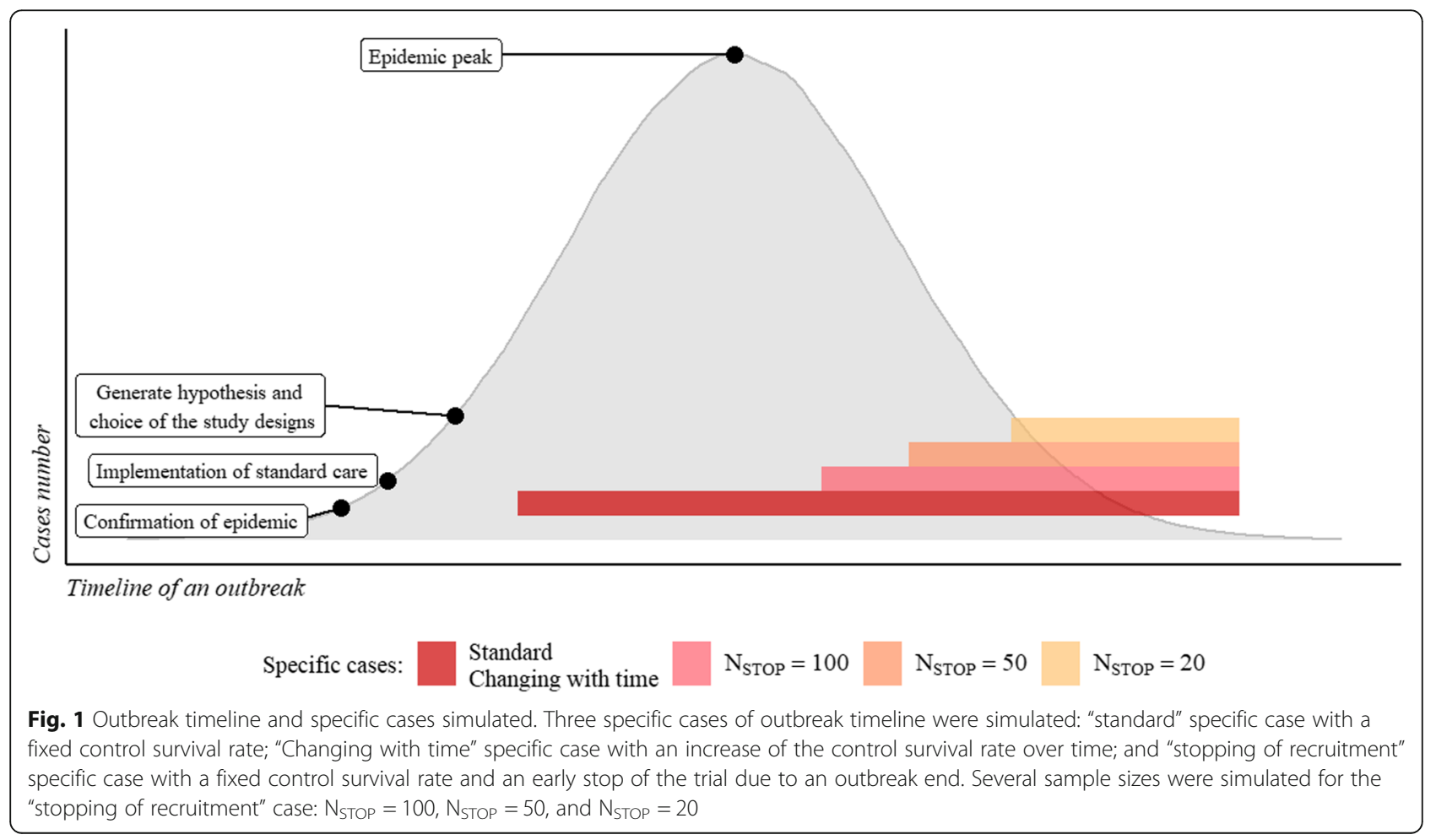


was extrapolated from the real weekly evolution of the survival rate during the 2014's Ebola epidemic in West Africa [17].

The "stopping of recruitment" case was performed to mimic the effect of starting recruitment at different times during the time course of the outbreak after the epidemic peak. Several sample sizes were simulated: $\mathrm{N}_{\mathrm{STOP}}=20, \mathrm{~N}_{\mathrm{STOP}}=50$, and $\mathrm{N}_{\mathrm{STOP}}=100$, and we assumed a fixed $\mathrm{p}_{\mathrm{C}}$ not changing with time. If required, the $p$-values in group-sequential designs were adjusted for underrunning (group-sequential trial which ended before that the stopping rule has been fulfilled) [18].

\section{Statistical tests}

For fixed designs (F1 and F2), the treatment efficacy was evaluated by a one-sided comparison test of proportions. A Yate's continuity correction was applied if needed. The experimental treatment was considered significantly superior if the $p$-value was inferior to 0.025 with the power $1-\beta=0.90$. For group-sequential designs ( $\mathrm{S} 1$ and S2), Whitehead triangular one-sided tests were conducted [19]. Stopping boundaries to conclude to the treatment efficacy were calculated using a type-I-error $\alpha=0.025$ and a power $1-\beta=0.90$. Group-sequential analyses were conducted every 20 patients included for both single and two-arm trials; and at each interim analysis the statistics $\mathrm{Z}$ (cumulative benefit of experimental treatment) and V (global information) from the Whitehead triangular test were calculated. The stopping rules for an interim analysis $j$ were then defined as follows:

- If $\mathrm{Z}_{\mathrm{j}} \geq \mathrm{a}+\mathrm{cV}_{\mathrm{J}}-0.583 \sqrt{\mathrm{V}_{\mathrm{j}}-\mathrm{V}_{\mathrm{j}-1}}$, the trial was stopped as the experimental treatment was shown to be significantly better than the control;

- If $\mathrm{Z}_{\mathrm{j}} \leq-\mathrm{a}+3 \mathrm{cV} \mathrm{V}_{\mathrm{j}}+0.583 \sqrt{\mathrm{V}_{\mathrm{j}}-\mathrm{V}_{\mathrm{j}-1}}$, the trial was stopped due to futility/inefficacy of the experimental treatment;

- Otherwise, the trial continued.

The stopping rules for the final analysis J were defined by:

- If $\mathrm{Z}_{\mathrm{J}} \geq \mathrm{a}+\mathrm{cV}_{\mathrm{J}}-0.583 \sqrt{\mathrm{V}_{\mathrm{J}}-\mathrm{V}_{\mathrm{J}-1}}$, the experimental treatment was considered as significantly better than the control;

- Otherwise, efficacy was not shown.

In case of premature termination of the groupsequential trial:

- If premature stopping of the trial occurred due to external reasons, i.e. independently of the cumulative information and the observed difference in efficacy between treatments, the final $p$-values were adjusted by the method proposed by Whitehead for underrunning [18],

- Otherwise, efficacy was not shown.

\section{Sample sizes}

The basic scenario was used to calculate the sample sizes. It assumed a "standard" case for the outbreak, a proportion of patient who survived at day $14 \mathrm{p}_{\mathrm{C}}=0.5$ (control survival rate) for two-arm trials, and $\mathrm{p}_{\mathrm{H}}=0.5$ (pre-trial historical survival rate) for single-arm trials, and an expected survival rate difference $\Delta=+0.2$. The number of subjects needed (NSN) for each fixed design and the maximum sample size for each group-sequential design $\left(\mathrm{N}_{\mathrm{Max}}\right)$, with a power $1-\beta=0.9$ and a one-sided type-I-error $\alpha=0.025$, were calculated and were: $\mathrm{NSN}_{\mathrm{F} 1}=60, \mathrm{NSN}_{\mathrm{F} 2}=248, \mathrm{~N}_{\mathrm{MAX}}, \mathrm{s} 1=91, \mathrm{~N}_{\mathrm{MAX}}, \mathrm{s} 2=378$.

For group-sequential trials, when the final analysis was performed with a cumulated number of subjects included equal to $\mathrm{N}_{\mathrm{Max}}$, the final analysis J was conducted with the remaining patients, necessarily inferior to 20 patients (number of patients included at each intermediate analysis).

Those sample sizes were used for all simulated scenario under "standard" or "changing with time" cases.

\section{Simulated values of parameters}

All specific cases of outbreak timeline and all designs were simulated with different values of the survival rate in the control arm $p_{C}=(0.35 ; 0.50 ; 0.75)$ and survival rate difference $\Delta=(-0.10 ; 0 ; 0.10 ; 0.20)$. Of note $\Delta=0$ corresponds to simulation under the null hypothesis. The experimental survival rate in the treatment arm was defined as $\mathrm{p}_{E}=\mathrm{p}_{C}+\Delta$. When $\mathrm{p}_{C}+\Delta \leq 0$ then $\mathrm{p}_{E}$ was set at 0.01 in order to be able to calculate the log-odds ratio for triangular tests. Likewise, $\mathrm{p}_{\mathrm{E}}$ was set at 1 when $\mathrm{p}_{\mathrm{C}}+$ $\Delta>1$.

For single-arm trials the fixed pre-trial historical survival rate $\mathrm{p}_{\mathrm{H}}$ was assumed to be 0.50 regardless of the scenario and control survival rate.

\section{Design evaluation}

For each design and each scenario, defined by the specific case of outbreak timeline and parameters $\mathrm{p}_{\mathrm{C}}$ and $\Delta$, $K=93,639$ simulated trials were simulated in order to ensure a $95 \%$ prediction interval width of 0.001 around $\alpha=0.025$. The seed was chosen randomly and was the same for all the scenarios. We set the seed once at the beginning of the entire set of simulated studies.

To evaluate the performance of each design, for each scenario, we calculated the proportion of simulated trials showing the efficacy of the experimental treatment i.e. the proportion of significant tests (denoted p), which corresponds to the type-I-error when $\Delta=0$, and the median [with 5th and 95th percentiles] number of subjects 
included in group-sequential trials (to be compared with the NSN of the fixed trials).

Simulations were performed with $\mathrm{R}$ software version 3.2.1.

\section{Results}

Scenarios under $\mathrm{H}_{0}(\Delta=0)$

For the basic scenario $\left(\mathrm{p}_{\mathrm{C}}=0.50\right.$ and $\left.\Delta=0\right)$, the type-Ierror was maintained regardless of the trial design (Table 1). For the "changing with time" case, the type-Ierror was calculated for one-arm designs exclusively: for two-arm designs, control and experimental survival rates increased together. Moreover, the type-I-error for singlearm designs $\mathrm{F} 1$ and $\mathrm{S} 1$ increased respectively to $p=$ 0.069 and $p=0.062$. This was due to the incorrect assumption on the pre-trial historical survival rate set at $\mathrm{p}_{\mathrm{H}}=0.50$ whereas the survival rate increased during the trial conduct, leading to a $\mathrm{p}_{\mathrm{H}}$ inferior to the control survival rate $\mathrm{p}_{\mathrm{C}}$. The assumption on the pre-trial historical survival rate is very important. Indeed, a wrong assumption on the value of the pre-trial historical survival rate $\left(\mathrm{p}_{\mathrm{H}}\right)$ has considerable impact on the results. For the "standard" and "changing with time" cases, when $\mathrm{p}_{\mathrm{C}}=$ 0.75 , whereas we assumed $\mathrm{p}_{\mathrm{H}}=0.50$, i.e, $\mathrm{p}_{\mathrm{H}}<\mathrm{p}_{\mathrm{C}}$, and $\Delta=0$, single-arm trials (either fixed or group-sequential) still had higher proportions of simulated trials showing a significant efficacy than two-arm trials. On the contrary, when $\mathrm{p}_{\mathrm{H}}>\mathrm{p}_{\mathrm{C}}$, with $\mathrm{p}_{\mathrm{C}}=0.35$ and $\Delta=0$, proportions of simulated trials showing a significant efficacy was very close to zero. Of note, for the "changing with time" case, when $\mathrm{p}_{\mathrm{C}}=0.75$ and $\Delta=0$, the type-I-error was lower for group-sequential two-arm design $(p=0.015)$ than fixed two-arm design $(p=0.025)$. This was due to the presence of $11 \%$ of inconclusive trials for the group-sequential design (data not shown).

Regarding the "stopping of recruitment" case, the only scenario where the type-I-error was maintained was observed under $\mathrm{H}_{0}$ for the group-sequential single-arm design with $\mathrm{p}_{\mathrm{C}}=0.50, \Delta=0$ and $\mathrm{N}_{\mathrm{STOP}}=100$, a sample size very close to the $\mathrm{N}_{\max , \mathrm{S} 1}$ (Table 2). When inclusions were poor and $\mathrm{p}_{C}=0.50, \Delta=0$, the type-I-error was always lower. For this specific case with $\mathrm{p}_{\mathrm{C}}=0.75, \Delta=0$ and $\mathrm{N}_{\mathrm{STOP}}=50$, wrong assumption on $\mathrm{p}_{\mathrm{H}}$ had a stronger impact on the type-I-error estimated at 0.95 to 0.97 for single-arm designs.

\section{Scenarios under $\mathrm{H}_{1}(\Delta=0.2)$}

For the "standard" case, proportions of simulated trials with a significant efficacy were similar $(p=0.90)$ between single- and two-arm designs under $\mathrm{H}_{1}$ when the control survival rate was equal to the pre-trial historical survival rate $\left(\mathrm{p}_{\mathrm{C}}=\mathrm{p}_{\mathrm{H}}=0.50\right)$ and $\Delta=0.2$ (Table 1$)$. However, when the pre-trial historical survival rate was incorrect, i.e. the control survival rate was lower $\left(\mathrm{p}_{C}=0.35\right)$ than the pre-trial historical survival rate, power of single-arm designs was poor. Indeed, only $11-12 \%$ of simulated single-arm trials were significant when $\mathrm{p}_{\mathrm{C}}=0.35$ and $\mathrm{p}_{\mathrm{E}}=0.55(\Delta=0.20)$, versus $88-89 \%$ of the simulated two-arm trials. For "changing with time" cases, proportions were overall similar between fixed and groupsequential designs (Table 1). Regarding "stopping of recruitment" cases, single-arm trials performed better than two-arm trials (Table 2). When $\mathrm{p}_{\mathrm{C}}=0.50$, the proportions of simulated trials showing a significant efficacy were higher for single-arm trials, ranging from $41 \%$ $\left(\mathrm{N}_{\mathrm{STOP}}=20\right)$ to $99 \%\left(\mathrm{~N}_{\mathrm{STOP}}=100\right)$, compared to twoarm trials (from $5 \%$ for $\mathrm{N}_{\mathrm{STOP}}=20$ to $54 \%$ for $\mathrm{N}_{\mathrm{STOP}}=$ 100). Moreover, fixed designs reported higher significant efficacy than group-sequential designs when $\Delta=0.20$.

\section{Scenarios with $\Delta=-0.1$}

When the treatment was harmful $(\Delta=-0.1)$, the proportion of simulated trials showing the efficacy of the

Table 1 Proportion of trials showing significant improvement with the experimental treatment ("standard" and "changing with time" cases)

\begin{tabular}{|c|c|c|c|c|c|c|c|c|c|}
\hline & \multirow[t]{2}{*}{ Design } & \multicolumn{4}{|l|}{$p_{C}=0.50$} & \multicolumn{2}{|l|}{$p_{C}=0.35$} & \multicolumn{2}{|l|}{$p_{C}=0.75$} \\
\hline & & $\Delta=0.20$ & $\Delta=0.10$ & $\Delta=0$ & $\Delta=-0.10$ & $\Delta=0.20$ & $\Delta=0$ & $\Delta=0$ & $\Delta=-0.10$ \\
\hline \multirow[t]{4}{*}{ Standard } & $\mathrm{F} 1$ & 0.896 & 0.351 & 0.025 & 0.0002 & 0.120 & 0.000 & 0.985 & 0.662 \\
\hline & S1 & 0.893 & 0.339 & 0.024 & 0.0003 & 0.115 & 0.0001 & 0.982 & 0.657 \\
\hline & F2 & 0.897 & 0.347 & 0.024 & 0.0001 & 0.887 & 0.025 & 0.025 & 0.0001 \\
\hline & S2 & 0.904 & 0.339 & 0.025 & 0.0004 & 0.883 & 0.025 & 0.024 & 0.0002 \\
\hline \multirow{4}{*}{$\begin{array}{l}\text { Changing } \\
\text { with time }\end{array}$} & F1 & 0.964 & 0.536 & 0.069 & 0.0011 & 0.241 & 0.0001 & 0.997 & 0.823 \\
\hline & $\mathrm{S} 1\left(^{\mathrm{a}}\right)$ & $0.944(0)$ & $0.520(0)$ & $0.062(0)$ & $0.0008(0)$ & $0.229(0)$ & $0(0)$ & $0.991(0)$ & $0.796(0)$ \\
\hline & F2 & 0.926 & 0.370 & 0.025 & 0.0002 & 0.875 & 0.023 & 0.025 & 0.0001 \\
\hline & $\mathrm{S} 2\left(^{\mathrm{a}}\right)$ & $0.958(0.0007)$ & $0.390(0.0001)$ & $0.024(0)$ & $0.0004(0)$ & $0.880(0)$ & $0.025(0)$ & $0.015(0.11)$ & $0(0)$ \\
\hline
\end{tabular}

In bold, type-l-errors maintained in [0.024;0.026]

Abbreviations: $\mathrm{p}_{\mathrm{C}}$ indicates control survival rate; $\Delta$ : simulated survival rate difference; F1: fixed single-arm design; S1: group-sequential single-arm design; F2: fixed two-arm design; S2: group-sequential two-arm design; Standard: specific case with a fixed control survival rate; Changing with time: specific case with an increase of the control survival rate over time

$\left({ }^{a}\right)$ : Proportion of significant tests with proportion of inconclusive group-sequential trials for "changing with time" case 
Table 2 Proportion of trials showing significant improvement for "stopping of recruitment" cases

\begin{tabular}{|c|c|c|c|c|c|c|c|c|}
\hline \multirow{2}{*}{$\begin{array}{l}\text { Stopping } \\
\text { of } \\
\text { recruitment }\end{array}$} & \multirow[t]{2}{*}{ Design } & & \multicolumn{3}{|l|}{$p_{c}=0.50$} & \multicolumn{3}{|l|}{$p_{C}=0.75$} \\
\hline & & & $\Delta=0.20$ & $\Delta=0$ & $\Delta=-0.10$ & $\Delta=0.20$ & $\Delta=0$ & $\Delta=-0.10$ \\
\hline \multirow[t]{6}{*}{$\mathrm{N}_{\mathrm{STOP}}=20$} & F1 & & 0.416 & 0.021 & 0.002 & 1 & 0.618 & 0.244 \\
\hline & S1 & $\left({ }^{\mathrm{a}}\right)$ & $0.414(0.75)$ & $0.021(0.587)$ & $0.002(0.245)$ & $1(0.003)$ & $0.615(0.585)$ & $0.242(0.830)$ \\
\hline & & $\left({ }^{b}\right)$ & $0.234+0.180$ & $0.006+0.015$ & $0.0004+0.0012$ & $0.997+0.002$ & $0.411+0.204$ & $0.116+0.126$ \\
\hline & $\mathrm{F} 2$ & & 0.054 & 0.006 & 0.002 & 0.048 & 0.005 & 0.001 \\
\hline & S2 & $\left({ }^{\mathrm{a}}\right)$ & $0.118(1)$ & $0.042(1)$ & $0.060(1)$ & $0.054(1)$ & $0.019(1)$ & $0.044(1)$ \\
\hline & & $\left({ }^{b}\right)$ & $0+0.118$ & $0+0.042$ & $0+0.060$ & $0+0.054$ & $0+0.019$ & $0+0.044$ \\
\hline \multirow[t]{6}{*}{$\mathrm{N}_{\mathrm{STOP}}=50$} & F1 & & 0.858 & 0.032 & 0.0005 & 1 & 0.972 & 0.619 \\
\hline & S1 & $\left({ }^{a}\right)$ & $0.793(0.136)$ & $0.023(0.020)$ & $0.0004(0.0002)$ & $1(0)$ & $0.948(0.054)$ & $0.526(0.181)$ \\
\hline & & $\left({ }^{b}\right)$ & $0.720+0.073$ & $0.016+0.007$ & $0.0003+0.0001$ & $1+0$ & $0.916+0.032$ & $0.438+0.088$ \\
\hline & $\mathrm{F} 2$ & & 0.333 & 0.032 & 0.005 & 0.320 & 0.021 & 0.003 \\
\hline & S2 & $\left({ }^{\mathrm{a}}\right)$ & $0.234(0.987)$ & $0.016(0.950)$ & $0.003(0.837)$ & $0.420(0.998)$ & $0.020(0.989)$ & $0.017(0.903)$ \\
\hline & & $\left(^{b}\right)$ & $0.013+0.221$ & $0.0001+0.016$ & $0+0.003$ & $0.002+0.418$ & $0+0.020$ & $0+0 ; 017$ \\
\hline \multirow[t]{5}{*}{$N_{\text {STOP }}=100$} & F1 & & 0.988 & 0.028 & 0 & 1 & 1 & 0.875 \\
\hline & S1 & & $0.894(0)$ & $0.025(0)$ & $0.0005(0)$ & $1(0)$ & $0.981(0)$ & $0.661(0)$ \\
\hline & $\mathrm{F} 2$ & & 0.542 & 0.029 & 0.002 & 0.834 & 0.024 & 0.0008 \\
\hline & S2 & $\left({ }^{a}\right)$ & $0.425(0.732)$ & $0.012(0.529)$ & $0.0004(0.190)$ & $0.747(0.765)$ & $0.012(0.766)$ & $0.0004(0.287)$ \\
\hline & & $\left({ }^{b}\right)$ & $0.255+0.170$ & $0.005+0.007$ & $0.0001+0.0003$ & $0.236+0.511$ & $0.001+0.011$ & $0+0.0004$ \\
\hline
\end{tabular}

In bold, type-l-errors maintained in [0.024;0.026]

Abbreviations: $\mathrm{p}_{\mathrm{C}}$ indicates control survival rate; $\Delta$ : simulated survival rate difference; F1: fixed single-arm design; S1: group-sequential single-arm design; F2: fixed two-arm design; S2: group-sequential two-arm design; Stopping of recruitment: specific case with a fixed control survival rate and an early stop of the trial due to an outbreak end; For group-sequential trials, the total of trials demonstrating an efficacy of the experimental treatment was defined by the sum of trials with a significant test and trials with an adjusted $p$-value inferior to 0.025 . The $p$-value was adjusted for underrunning using the method proposed by Whitehead [18] ${ }^{a}$ ): Proportion of significant tests with proportion of inconclusive group-sequential trials for "changing with time" cases

$\left({ }^{b}\right)$ : Proportion of significant tests + proportion of trials with adjusted $p$-values inferior to 0.025 for group-sequential trials and "stopping of recruitment" cases

experimental treatment was close to 0 regardless of the specific case, except when $\mathrm{p}_{\mathrm{C}}=0.75$ for single-arm designs, which was due to the wrong assumption on $\mathrm{p}_{\mathrm{H}}$ (Tables 1 and 2).

\section{Number of subjects included}

The median number $\left[\mathrm{P}^{\text {th }}-\mathrm{P} 95^{\text {th }}\right]$ of subject included in group-sequential trials for both "standard" and "changing with time" cases are reported in Fig. 2 with $\mathrm{p}_{\mathrm{C}}=0.50$. The median number of included subjects was always lower in the group-sequential trials compared with the fixed trials, especially when the experimental treatment effect was harmful $(\Delta=-0.10)$. Indeed, scenarios with $\Delta=-0.10$ or $\Delta=0$ always presented a median number of included subjects lower than scenarios with $\Delta>0$. Moreover, less than $10 \%$ of the group-sequential single- or two-arm trials reported a higher number of included subjects than single- or two-arm fixed trials respectively (Table 3). On the other hand, the NSN and $\mathrm{N}_{\text {Max }}$ determined for two-arm designs were greater to 200 patients whereas the NSN and $\mathrm{N}_{\text {Max }}$ for single-arm designs were lower to 100 patients. The rapidity to perform a singlearm trial compared with two-arm designs, which require more than the double of inclusions, is considerable.

\section{Online tool to visualize results of simulated scenarios}

Simulation results were reported online on the following dashboard: https://pmn-bch.shinyapps.io/simu-vhf/. This tool reports all scenarios for all designs (F1, F2, S1, S2), according to the choice of parameters: the control survival rate $\mathrm{p}_{\mathrm{C}}$ and the survival rate difference $\Delta$. Available values are, for $\mathrm{p}_{\mathrm{C}}: 0.35,0.40,0.45,0.50,0.65,0.75$, and for $\Delta:-0.40,-0.20,-0.10,0,0.10,0.20,0.30,0.40$. Several specific cases of an outbreak can be selected: the "standard" one (with fixed $\mathrm{p}_{\mathrm{C}}$, the NSN for fixed designs and $\mathrm{N}_{\mathrm{Max}}$ if required for group-sequential trials), the "changing with time" one which simulated the evolution of the survival rate and the "stopping of recruitment" which simulated an early stop of the trial (with $\mathrm{N}_{\mathrm{STOP}}=$ $20 ; \mathrm{N}_{\mathrm{STOP}}=50 ; \mathrm{N}_{\mathrm{STOP}}=100$ ). This tool will allow to evaluate all the elements before designing a clinical trial in the context of an outbreak of a viral haemorrhagic fever.

\section{Discussion}

We presented results from our simulation to help future investigators choosing the best clinical trial design (single- versus two-arm, fixed versus group-sequential trial) 


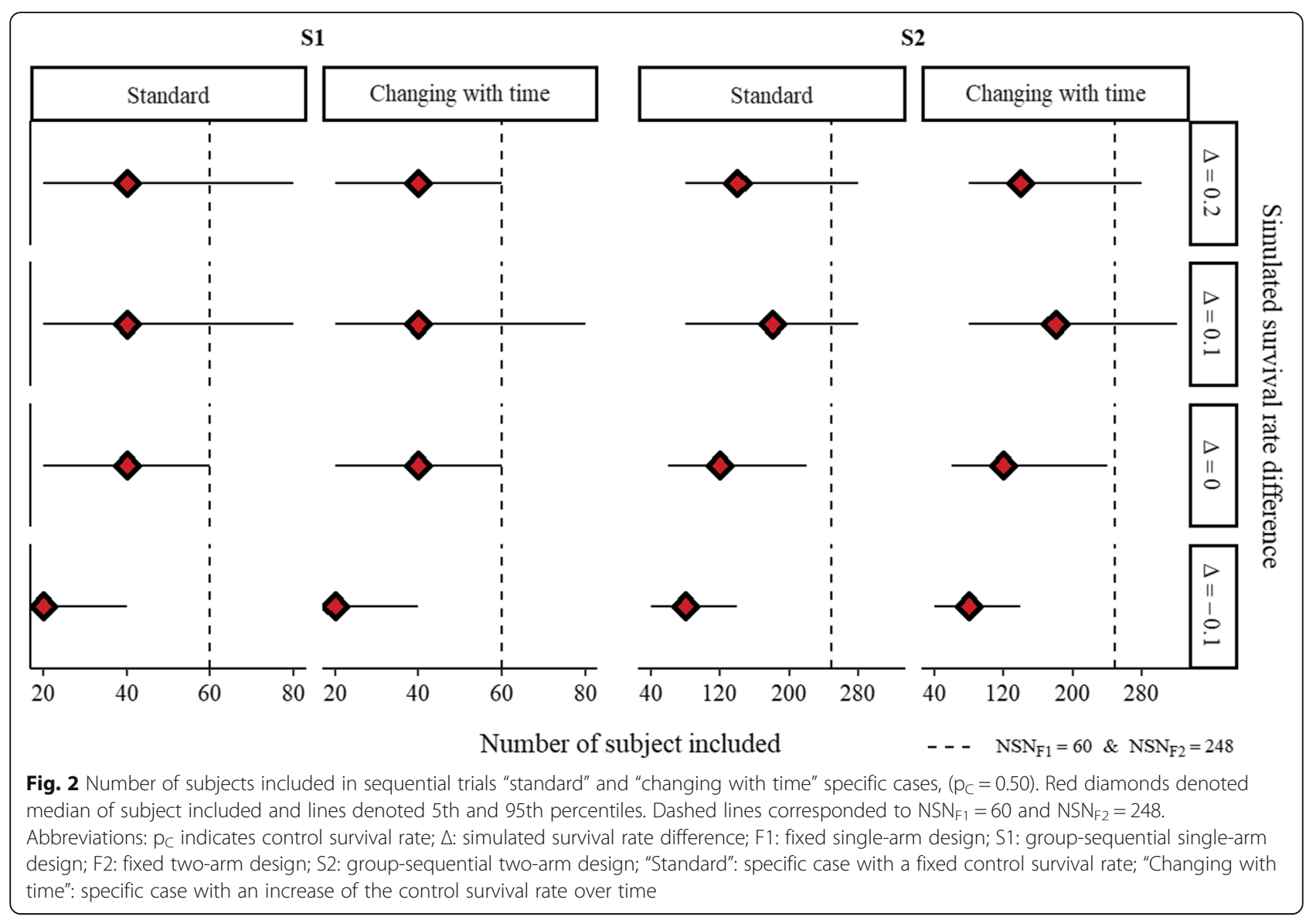

based on the outbreak timeline of an emerging infectious disease.

At the beginning of an outbreak group-sequential twoarm trials should be preferred. A group-sequential design will often allow an early termination of the trial when the treatment does not perform better than the control or when treatment efficacy is large. Moreover, the number of infected cases increases until the epidemic peak. Thus, the required number of patients to perform a two-arm trial can be reached and the

Table 3 Proportion of group-sequential trials with subjects numbers superior to fixed designs NSN ( $\left.p_{C}=0.50\right)$

\begin{tabular}{llllll}
\hline & & $\boldsymbol{\Delta}=\mathbf{0 . 2}$ & $\boldsymbol{\Delta}=\mathbf{0 . 1}$ & $\boldsymbol{\Delta}=\mathbf{0}$ & $\boldsymbol{\Delta}=\mathbf{- 0 . 1}$ \\
\hline Standard & S1 & 0.07 & 0.13 & 0.02 & 0.0004 \\
& S2 & 0.09 & 0.15 & 0.02 & 0.0003 \\
Changing & S1 & 0.02 & 0.13 & 0.04 & 0.001 \\
with time & S2 & 0.1 & 0.23 & 0.03 & 0.0005 \\
\hline
\end{tabular}

Abbreviations: $\mathrm{p}_{\mathrm{C}}$ indicates control survival rate; $\Delta$ : simulated survival rate difference; F1: fixed single-arm design; S1: group-sequential single-arm design; F2: fixed two-arm design; S2: group-sequential two-arm design; "Standard": specific case with a fixed control survival rate; "Changing with time": specific case with an increase of the control survival rate over time potential evolution of the survival rate will be considered with the presence of the control arm.

For trials beginning after the epidemic peak fixed single-arm design should be performed. As the number of cases decreases after the peak of the epidemic, singlearm designs would be preferred as they required a lower number of patients than two-arm designs. However, this assumes that the pre-trial historical survival rate was correctly estimated. We showed that with an incorrect assumption on this survival rate, the type-I-error is not maintained. Single-arm trials should only be conducted if a high level of confidence can be put on the pre-trial historical survival rate used.

From a methodological standpoint, concern must be taken when conducting a group-sequential trial. Indeed, for "stopping of recruitment" case presenting a premature termination due to an epidemic end, the adjustment for underrunning had an important impact on the results. The number of simulated trials showing a significant efficacy increased after adjusting for underrunning: the adjusted $p$-value led to conclude more often to efficacy of treatment (Fig. 3). Therefore, in the case of an epidemic end, where the cumulative information for group-sequential trials is too low due to the impossibility 


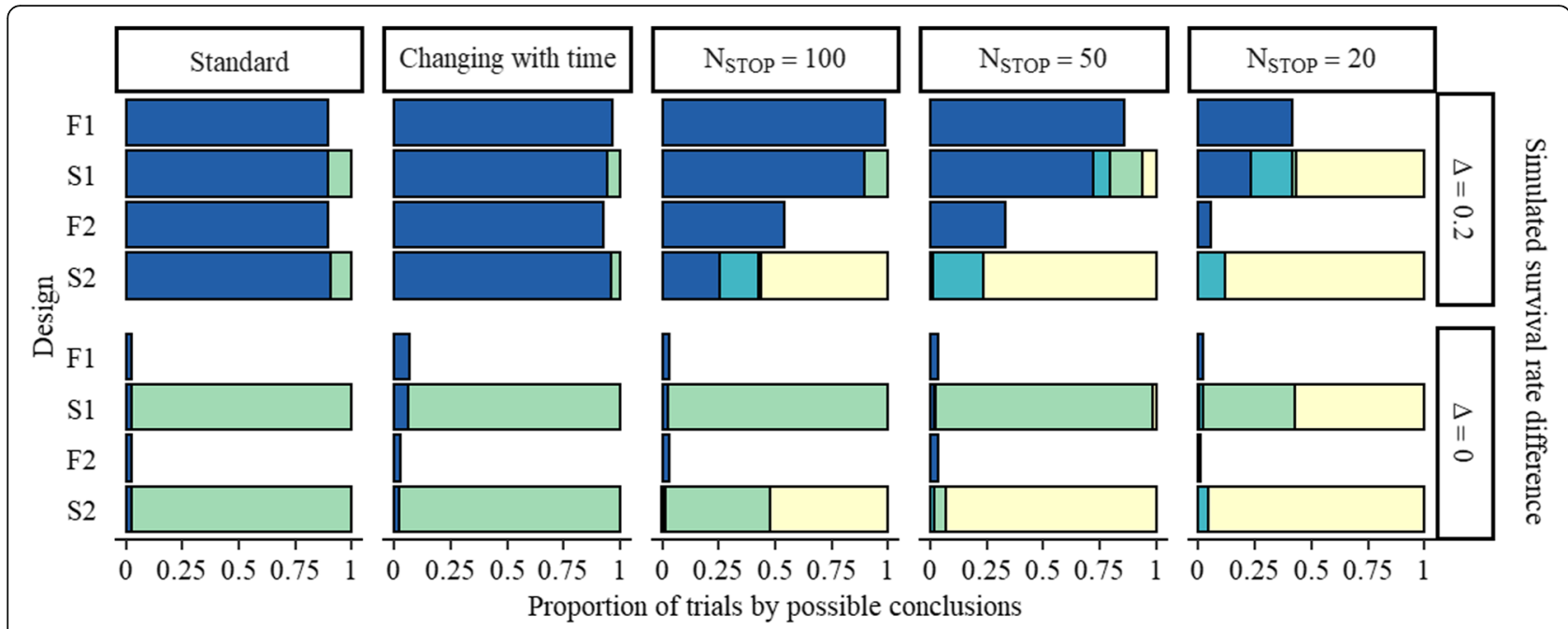

$\square$ Efficacy $\square$ Efficacy with significant level adjusted $\square$ Futility $\square$ Inconclusive

Fig. 3 Proportion of significant tests with distribution of conclusions for each design and specific case $\left(p_{C}=0.50\right)$. Abbreviations: $p_{C}$ indicates control survival rate; $\Delta$ : simulated survival rate difference; F1: fixed single-arm design; S1: group-sequential single-arm design; F2: fixed two-arm design; S2: group-sequential two-arm design; "Standard": specific case with a fixed control survival rate; "Changing with time": specific case with an increase of the control survival rate over time; "Stopping of recruitment": specific case with a fixed control survival rate and an early stop of the trial due to an outbreak end. For group-sequential trials, the total of trials demonstrating an efficacy of the experimental treatment was defined by the sum of trials with a significant test and trials with an adjusted $p$-value inferior to 0.025. The $p$-value was adjusted for underrunning by the method proposed by Whitehead [18]

of including patients, it is necessary to consider the adjusted $p$-value to limit the loss of information and to increase the probability to conclude (for futility or efficacy).

One limitation of our study is that our recommendation on using single-arm trial if it starts after the epidemic peak is based on the assumption made on the pre-trial historical survival rate used to design the trial. Indeed, we noted that results are substantially biased when this value is incorrect and the treatment is harmful. Double-blind randomised controlled trial is the best method to use to avoid those erroneous conclusions. However, conducting a randomised controlled trial is not always feasible. In those cases, single-arm trials should be used when there is relatively strong evidence behind the pre-trial historical survival rate used. Another limitation is that our analyses are based on the widely used $2.5 \%$ threshold for the type-I-error, for a one-sided test. However, in the context of viral haemorrhagic fever, where mortality rates are high and few effective therapies are available, a $2.5 \%$ threshold may not be appropriate and may even raise ethical questions. This threshold has previously been challenged, and recent studies, especially in oncology, explored new methods (Bayesian Decision Analysis) to choose suitable thresholds that minimize the overall expected harm to patients within clinical trials and future patients especially for the deadliest diseases [20]. In this clinical trial simulation study, we decided to set the type-I-error at $2.5 \%$ for a one-sided test. By increasing the type-I-error, it is expected that the number of subjects included will decrease. However, the choice of this threshold is questionable. In particular, it could depend on the disease being studied, the potential outbreak context, the mortality rate, as well as the prevalence (e.g. rare disease).

An extension of this work would be to consider the case of multi-arm clinical trials. In the context of an outbreak, several treatments could be candidates. This was observed for Ebola virus, where various treatments were tested during the same outbreak period [6-8, 12, 13]. Each treatment was evaluated by one clinical trial and associated with a specific research institute and country. Multi-arm trial has the advantage of reducing the number of subjects to be included: indeed, a single control arm is needed against different experimental arms without loss of power. Launching several two-arm trials increases the number of control arms and the global sample size to find the right treatment [21, 22]. It also raises difficulties about acceptability for the participants and lead to a large loss of time due to competing clinical trials. The gain in the number of subjects is an important point in the context of an outbreak where the number of cases decreases following its peak and the choice of treatment to be tested is uncertain. Moreover, group- 
sequential trials allow to minimise the expected number of subjects included regardless of the number of arms in the trial design. The same considerations explored in this simulation study for two-arm trials would apply to multi-arm trials; except for the case of group-sequential multi-arm design, where after premature termination of treatment arms for futility, more patients would have the possibility to receive the remaining treatment. Comparing the performance of a multi-arm group-sequential trial to a fixed trial at the end of an outbreak would be interesting, but would require to explore a multitude of scenarios, such as the efficacy or futility of each treatment.

In this simulation study, we decided to evaluate a randomization 1:1 for two-arm trial designs. An unequal allocation ratio in favour of the experimental treatment arm would have increased the probability of treatment exposure of the included patient. At first sight, this strategy is beneficial, especially at the end of an outbreak when the number of cases and the number of inclusions is decreasing substantially. However, the loss of power due to this mode of randomization is not negligible: despite an increase in the proportion of patients receiving treatment, this design would therefore require larger sample sizes to achieve the same level of statistical power [23].

Furthermore, this point questions the choice of designs for group-sequential rather than adaptive trials. This type of trial is based on the same principles as group-sequential trials: to adapt the trial based on data accumulated during the study. For example, in a groupsequential trial, a treatment arm will be stopped for futility. With an adaptive trial, it will be possible to reevaluate during the study the number of subjects needed and change the probability of assigning a treatment (as in the case of unequal allocation ratios).

In practice, adaptive trials are mainly used for the evaluation of treatment doses. They allow, like multi-stage multi-arm trials (MAMS), the combination of phase II and phase III trials. Our simulation study focuses on the performance of phase III clinical trials. Further work could be conducted on adaptive methodologies and MAMS. New questions arise concerning the efficiency of these methods compared with phase II and III clinical trials, with the aim of accelerating the therapeutic evaluation process, using both Bayesian and frequentist approaches $[15,24]$. In the case of a VHF outbreak, such as Ebola virus, one MAMS phase II - phase III trial was conducted with a multicentre, multi-outbreak, randomized controlled trial design [25]. In addition, by focusing on adaptive phase III clinical trials, the reasoning would no longer be short-term (a single outbreak wave) but medium and even long-term with withdrawal and/or addition of treatment arms according to the evolution of knowledge about the disease studied, eligible treatments, and marketing authorisations specific to each country. Indeed, an adaptive trial, a MAMS trial, or a classic multi-arm trial seem to be possible over several outbreak waves (multi-outbreak).

Following this work, we wish to create a more complete Shiny App were users could enter other values than that presently available in the drop-down menus.

\section{Conclusion}

In conclusion, the choice of the clinical trial design to be conducted depends on the timeline of the outbreak of a viral haemorrhagic fever. At the beginning of the outbreak, group-sequential two-arm trials should be preferred, as the number of infected cases will increase until the epidemic peak allowing to conduct a strong randomised controlled trial. Moreover, a group-sequential design will allow an early termination of the trial in cases of harmful experimental treatment. The stopping for futility would be faster than the stopping for efficacy, which is an important aspect during first period of an outbreak, usually corresponding to a treatment screening phase.

Regarding trials beginning after the epidemic peak, fixed single-arm design should be performed, as the number of cases decreases after the peak of the outbreak, reducing the number of patients that could be included. However, this assumes that a high level of confidence can be put on the pre-trial historical survival rate used in the single-arm trial.

\section{Abbreviations}

F1: Fixed single-arm design; F2: Fixed two-arm design; MSA: Multi-stage approach; NSN: Numbers of subject needed; $\mathrm{p}_{c}$ : Control survival rate, the proportion of patient who survived in the control arm; $\mathrm{p}_{\mathrm{E}}$ : Experimental survival rate, the proportion of patient who survived in the experimental arm; $\mathrm{p}_{\mathrm{H}}$ : Pre-trial historical survival rate; S1: Group-sequential single-arm design; S2: Group-sequential two-arm design

\section{Supplementary Information}

The online version contains supplementary material available at https://doi. org/10.1186/s12874-021-01287-w.

Additional file 1. Description of the assessed clinical trial designs.

\section{Acknowledgements}

Not applicable.

\section{Authors' contributions}

PM, FM and $C L$ designed the simulation study. PM performed the simulation and drafted the manuscript. PM, FM and CL participated in the statistical analysis and DB helped to draft the manuscript. All authors read and approved the final manuscript.

\section{Funding}

Not applicable.

\section{Availability of data and materials}

The datasets used and/or analysed during the current study are available from the corresponding author on reasonable request. The $\mathrm{R}$ codes for simulations are available in GitHub: https://github.com/PMN-BCH/simu-VHF. 
All results are available were in the online Shiny App: https://pmn-bch. shinyapps.io/simu-vhf/.

\section{Declarations}

Ethics approval and consent to participate

Not applicable.

\section{Consent for publication}

Not applicable.

\section{Competing interests}

The authors declare that they have no competing interests.

\section{Author details}

${ }^{1}$ INSERM, Centre d'Investigation clinique-Epidémiologie Clinique 1425, Hôpital Bichat, F-75018 Paris, France. ²Département Epidémiologie Biostatistiques et Recherche Clinique, AP-HP, Hôpital Bichat, F-75018 Paris, France. ${ }^{3}$ Université de Paris, INSERM, IAME UMR 1137, F-75018 Paris, France.

Received: 2 November 2020 Accepted: 21 April 2021

Published online: 06 May 2021

\section{References}

1. World Health Organization. Disease outbreaks. http://www.who.int/ emergencies/diseases/en/. Accessed 24 Jan 2020.

2. World Health Organization. Middle East respiratory syndrome coronavirus (MERS-CoV). WHO. http://www.who.int/emergencies/mers-cov/en/. Accessed 22 Oct 2020

3. Centers for Disease Control and Prevention, National Center for Emerging and Zoonotic Infectious Diseases (NCEZID), Division of High-Consequence Pathogens and Pathology (DHCPP), Viral Special Pathogens Branch (VSPB). https://www.cdc.gov/vhf/index.html. Accessed 1 Jul 2019

4. Ebola health update - North Kivu/Ituri, DRC, 2018-2020. https://www.who. int/emergencies/diseases/ebola/drc-2019. Accessed 22 Oct 2020.

5. Sigfrid L, Maskell K, Bannister PG, Ismail SA, Collinson S, Regmi S, et al. Addressing challenges for clinical research responses to emerging epidemics and pandemics: a scoping review. BMC Med. 2020;18(1):190. https://doi.org/10.1186/s12916-020-01624-8.

6. PREVAIL II Writing Group, Multi-National PREVAIL II Study Team, Davey RT, Dodd L, Proschan MA, Neaton J, et al. A Randomized, Controlled Trial of ZMapp for Ebola Virus Infection. N Engl J Med. 2016;375:1448-56.

7. Sissoko D, Laouenan C, Folkesson E, M'Lebing A-B, Beavogui A-H, Baize S, et al. Experimental treatment with Favipiravir for Ebola virus disease (the JIKI trial): a historically controlled, Single-Arm Proof-of-Concept Trial in Guinea. PLoS Med. 2016;13:e1001967.

8. van Griensven J, Edwards T, de Lamballerie X, Semple MG, Gallian P, Baize S, et al. Evaluation of convalescent plasma for Ebola virus disease in Guinea. N Engl J Med. 2016;374(1):33-42. https://doi.org/10.1056/NEJMoa1511812.

9. World Health Organization. Guidance for managing ethical issues in infectious disease outbreaks. World Health Organization. 2016. https://apps. who.int/iris/handle/10665/250580. Accessed 1 Jul 2019.

10. Adebamowo C, Bah-Sow O, Binka F, Bruzzone R, Caplan A, Delfraissy J$F$, et al. Randomised controlled trials for Ebola: practical and ethical issues. Lancet. 2014;384(9952):1423-4. https://doi.org/10.1016/S0140-673 6(14)61734-7.

11. Chow S-C, Chang M. Adaptive design methods in clinical trials - a review. Orphanet J Rare Dis. 2008:3(1):11. https://doi.org/10.1186/1750-1172-3-11.

12. Dunning J, Kennedy SB, Antierens A, Whitehead J, Ciglenecki I, Carson G, et al. Experimental treatment of Ebola virus disease with Brincidofovir. PLoS One. 2016;11(9):e0162199. https://doi.org/10.1371/journal.pone.0162199.

13. Dunning J, Sahr F, Rojek A, Gannon F, Carson G, Idriss B, et al. Experimental treatment of Ebola virus disease with TKM-130803: a single-arm phase 2 clinical trial. PLoS Med. 2016;13(4):e1001997. https://doi.org/10.1371/journal. pmed.1001997.

14. Mulangu S, Dodd LE, Davey RT, Tshiani Mbaya O, Proschan M, Mukadi D, et al. A randomized, controlled trial of Ebola virus disease therapeutics. N Engl J Med. 2019;381(24):2293-303. https://doi.org/10.1056/NEJMoa1910993.

15. Cooper BS, Boni MF, Pan-ngum W, Day NPJ, Horby PW, Olliaro P, et al. Evaluating clinical trial designs for investigational treatments of Ebola virus disease. PLoS Med. 2015;12(4):e1001815. https://doi.org/10.1371/journal. pmed.1001815.

16. Whitehead J, Olliaro P, Lang T, Horby P. Trial design for evaluating novel treatments during an outbreak of an infectious disease. Clin Trials. 2016; 13(1):31-8. https://doi.org/10.1177/1740774515617740.

17. Centers for Disease Control and Prevention. 2019. https://www.cdc.gov/vhf/ ebola/history/2014-2016-outbreak/case-counts.html. Accessed 26 Dec 2019.

18. Whitehead J. Overrunning and underrunning in sequential clinical trials. Control Clin Trials. 1992;13(2):106-21. https://doi.org/10.1016/0197-2456(92 )90017-T.

19. Whitehead J. The design and analysis of sequential clinical trials. Chichester: Wiley; 1997. ISBN: 978-0-471-97550-2.

20. Montazerhodjat V, Chaudhuri SE, Sargent DJ, Lo AW. Use of Bayesian decision analysis to minimize harm in patient-centered randomized clinical trials in oncology. JAMA Oncol. 2017;3(9):e170123. https://doi.org/10.1001/ja maoncol.2017.0123.

21. Freidlin B, Korn EL, Gray R, Martin A. Multi-arm clinical trials of new agents: some design considerations. Clin Cancer Res. 2008;14(14):4368-71. https:// doi.org/10.1158/1078-0432.CCR-08-0325.

22. Parmar MKB, Carpenter J, Sydes MR. More multiarm randomised trials of superiority are needed. Lancet. 2014;384(9940):283-4. https://doi.org/10.101 6/S0140-6736(14)61122-3.

23. Hey SP, Kimmelman J. The questionable use of unequal allocation in confirmatory trials. Neurology. 2014;82(1):77-9. https://doi.org/10.1212/01. wnl.0000438226.10353.1c.

24. Chevret S. Bayesian adaptive clinical trials: a dream for statisticians only? Stat Med. 2012;31(11-12):1002-13. https://doi.org/10.1002/sim.4363.

25. National Institute of Allergy and Infectious Diseases (NIAID). A Multicenter, Multi-Outbreak, Randomized, Controlled Safety and Efficacy Study of Investigational Therapeutics for the Treatment of Patients With Ebola Virus Disease. Clinical trial registration. clinicaltrials.gov; 2020. https://clinicaltrials. gov/ct2/show/study/NCT03719586. Accessed 24 Jan 2021.

\section{Publisher's Note}

Springer Nature remains neutral with regard to jurisdictional claims in published maps and institutional affiliations.

Ready to submit your research? Choose BMC and benefit from:

- fast, convenient online submission

- thorough peer review by experienced researchers in your field

- rapid publication on acceptance

- support for research data, including large and complex data types

- gold Open Access which fosters wider collaboration and increased citations

- maximum visibility for your research: over $100 \mathrm{M}$ website views per year

At BMC, research is always in progress.

Learn more biomedcentral.com/submission 